\title{
A Framework for Adoption and Diffusion of Mobile Applications in Africa
}

\author{
Chinedu Wilfred Okonkwd ${ }^{7} 1,2$, Magda Huisman ${ }^{2}$, Estelle Taylor ${ }^{2}$ \\ ${ }^{1}$ School of Consumer Intelligence and Information Systems, University of Johannesburg, Johannesburg, 2001, South Africa \\ ${ }^{2}$ School of Computer, Statistical and Mathematical Sciences, North-West University, Potchefstroom Campus, 2520, South Africa
}

\section{A R T I C L E INFO}

Article history:

Received: 11 October, 2020

Accepted: 28 November, 2020

Online: 25 December, 2020

\section{Keywords:}

Mobile Applications

Adoption

Diffusion

Framework

Africa

\begin{abstract}
A B S T R A C T
The adoption and diffusion of mobile applications (mobile apps) has become the base of modern activities in Africa owing to the services and values that are obtained through mobile apps innovations. More emphasis has been on the development of mobile apps whereas the adoption and diffusion process as well as their predictors are ignored or given less attention. This study explores and develops a framework for the adoption and diffusion of mobile apps in Africa. A survey was conducted on the basis of the diffusion of innovation framework in five-selected African nations. A total of 1285 of the 2300 distributed questionnaires were returned, giving a response rate of 55.87\%. The results indicated that many factors/predictors drive the acceptance and use of mobile apps and these factors were structured to develop a common framework for mobile apps adoption and diffusion in Africa.
\end{abstract}

\section{Introduction}

The advancement in mobile technology has had a major impact on the manner in which business and personal activities are performed. It could be said, in particular, that a radical evolution started with the advent of handheld devices such as mobile phones that caused the creation of distinct kinds of mobile apps that are used in separate activities [1, 2]. A number of mobile technology start-ups have been created in Africa to encourage mobile app innovation [2, 3] Advances in digital technology have enhanced communication systems in Africa, leading to a growing amount of African communities linked to digital communications [4, 5]. The use of mobile apps are changing the African society, socially, economically and politically [1], [6, 7]. Despite the penetration and growth of mobile services, some mobile apps are rejected and discontinued to be used. Moreover, there are some non-users of mobile apps in Africa [8]. The process of acquiring and disseminating mobile app services in Africa must therefore be improved, particularly for those with local content.

It is imperative to information and communication technology (ICT) experts and scholars to know the flow of information technologies [3]. This can be achieved by conducting a research on adoption and diffusion, which implies accepting to use [2, 9, 10] and continuing the use of a new innovation [11,12]. Various models have been proposed to aid this process including frameworks for mobile health [13], mobile banking [14] and e-government [15]. These models dealt on a particular mobile app in a specific country and cannot be generalised.

However, African mobile consumers are growing interest in home-grown mobile apps. Several mobile apps have originated in Africa, especially in South Africa, Nigeria and Kenya including social networking mobile apps like Mxit, 2go and How Far, entertainment mobile apps like Simfy Africa, Spinlet, iRokoTV and SOLO, and financial mobile apps like M-pesa [2]. These mobile apps are mostly used in the country where they are created whereas in the other countries of the continent, there is little or no adoption of the mobile apps. In other words, there is a significant difference between the rate of adoption of each respective mobile app between the country where it is created and the other countries in Africa.

This study proposes a general framework for mobile apps adoption and diffusion in African social system. Drawing from the literature on the adoption of mobile app innovation and deliveries, this research intends to address the following questions:

1. What are the factors that influences mobile apps adoption in

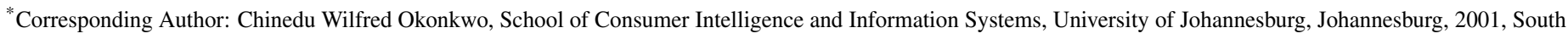
Africa., +27835796557 \& Email: chinedung@yahoo.com
} 


\section{Africa and}

2. How can we structure these factors to form a general model to enhance the acceptance and use of mobile app innovation in Africa.

In order to achieve these goals, we conducted a survey in the five countries representing the five geographical locations in Africa to assess the user's view of mobile app use in order to explore the factors predicting the acceptance and use of mobile apps in Africa. These factors were structured to develop a framework. With leverage on the report that there is a need to know the diffusion of mobile technologies and the important of mobile technological services on developing nations such as African countries, this study would therefore make the following contributions. We have:

1. Examined and discovered the acceptance and use of mobile apps in Africa,

2. determined the factors/predictors that influence users' intention to adopt and use mobile apps innovations in Africa, and

3. developed a general framework that can improve the adoption and use of different kinds of mobile apps across African nations.

The remainder of this research work is organised as follows: Section 2 discusses the background information of the research work. Section 3 describes the related works and the gap. Section 4 shows the research conceptual model and Section 5 explains the research methodology. Section 6 describes the methods of analysis and Section 7 zooms into the survey results. Section 8 presents the regression analysis and Section 9 discusses analysis of variance (ANOVA) results. Section 10 interprets the results to validate the proposed framework. Section 11 concludes the study.

\section{Background information}

A brief theoretical overview of the study is presented in this part.

\subsection{What are mobile apps?}

Mobile app creativity has recently attracted scholars from different fields of study to carry out studies in their respective fields. As a consequence, mobile apps have been described by several writers, write-ups and web pages in many perspectives including Technology [16, 17], Educational [18], Context-aware Computing [19], Internet of Things [20], Business [21], Cloud Computing [22] and Social Services [23]. In this study, mobile apps are considered to be mobile technological innovations of a mobile computer software that allows users to carry out various activities including political, social, economic, and educational regardless of the user's location and background.

\subsection{Features of mobile apps}

There are five main characteristics of mobile apps, according to Rogers [9]. They are relative advantage,compatibility, complexity, observability and trialability. Such characteristics help to decrease the level of misunderstanding and increase the adoption rate of mobile apps. Technology reliability and cost are conceptualized in this study as important features of mobile apps [8].

\subsection{Innovation decision process}

This is a process of decision-making which includes an informationseeking and information-processing practices in which mobile app adopters are driven to reduce the confusion surrounding the effect of acceptance and use of a new mobile app innovation. Rogers's diffusion framework consists of five stages of the innovation decision process including knowledge, persuasion, decision-making, implementation and confirmation [9]. For this study implementation and confirmation are merged because they describe diffusion (putting into use and continue to use) and referred to as the diffusion stage. Thus, the innovation decision process of this research entails four phases including knowledge, persuasion, decision-making and diffusion. These stages are briefly discussed below.

Knowledge stage:The mobile apps adoption and diffusion begins with the knowledge about mobile apps. This stage involves acquisition, method and sharing of information about mobile apps. Having the right knowledge about mobile apps requires information sources, learning methods and information sharing. In Africa, there are two distinct sources of information about mobile apps including external initiated actions and personal initiated actions, learning methods are interpersonal communications, the Internet, operational manual and informal training, whereas information sharing involves organizations and events, and individual activities [8].

Persuasion stag: is shaping the minds of adopters towards adopting and using mobile apps. The main factor under this stage is the social influence [9]. Decision-making stage: This includes the adoption and rejection of mobile apps. In this stage, an individual/adopter of mobile apps chooses to adopt or reject the mobile apps. The adoption covers the adopter's category whereas rejection covers the reasons for non-adoption [9]. There are five categories of adopters in Africa including late majority and laggards, nonadopters, innovators, early adopters and early majority [8]. Also, the reasons for non-adoption of mobile apps in Africa are using mobile apps is time-consuming, using mobile apps takes a lot of phone memory, using mobile apps distracts attention from other activities and using mobile is expensive [8]. Diffusion stage: This involves the implementation and confirmation. That is putting mobile apps into use and continuing to use them. It covers the use of mobile apps, ways of using mobile apps and reasons for discontinuance of the use of mobile apps. There are two distinct ways of using mobile apps in Africa including business use and individual use, and the reasons for discontinuance include a better replacement, unsatisfactory performance, High complexity and lack of support [8].

\subsection{Adopting Unit}

The adopting unit consists of features of individual adoption, comprising personality variables, socioeconomic and communication behavior. [9].

Personality variables: Personality variables entail the individual characteristics (demographic) that are part of the factors influenc- 
ing the outcome of the innovation decision process [9]. Adopters' attitudes, anxiety (degree of nervousness) and self-efficacy are conceptualized as part the personality variables. Attitude is measured by the feelings and reactions of the participants on the importance and contributions of mobile apps in Africa [24].

Socioeconomic characteristics: Socioeconomic characteristics involve the external factors that may influence individuals' adoption and diffusion of mobile apps. These characteristics include the contributions of mobile apps to the development of Africa [25], facilitating conditions and employment status[8] .

Communication behaviour: This covers the primary purpose of using and disseminating the information about a newly developed mobile app [9]. It is one of the primary goals that encourages a person to embrace and disseminate mobile apps. In this study, It deals with the function of information sharing as specified in the abstraction model of the research. This research deals with two key reasons of spreading the information about a newly created mobile app including: [8].

1. to facilitates both individual and business activities, and

2. to improve and make communications easier.

\subsection{Adoption}

According to Rogers [9], adoption is the absolute willingness to embrace and use new advanced technologies (mobile apps). GSMA [2] explained that adoption is embracing and using a new technology. It is the acceptance [10] and use [11] of an emerging product such as mobile apps by the consumer. Innovation is new when it is first introduced to a person. Therefore, we describe the adoption as the users' acceptance and use of a new invention.

\subsection{Diffusion}

Diffusion is a means of spreading the information and use of an innovation among the individuals of a social structure across certain networks [9, 3]. It is a mechanism by which a commodity is spread from one customer to another [11]. For this research, with regards to Rogers framework, we combine the implementation and confirmation phases to form diffusion. Thus, we defined diffusion as the method of sharing, uses and discontinued the uses of a product.

\subsection{Usage of mobile applications in Africa}

GSMA [26] recorded that, there are more than five billion mobile subscribers across the globe and the number is expected to rise to 5.9 billion by the year 2025 . The spread of mobile technology is wider than any other technology [27]. Mobile service penetration is expanding at an extraordinary rate in Africa, and mobile apps are part of the main foundations of all mobile services. The introduction of mobile services in Africa is rapidly growing such that the mobile app is the driving force behind some of the emerging developments in African nations. [16, 7]. The African continent currently ranks among the world's largest adopters of mobile technology services, introducing new mobile services to the population [28, 29].
The beginning of mobile app development can be linked to the program required to operate the emerged mobile devices [30]. Over the years, telecommunications in Africa was primarily through a fixed-line system and the networks were regulated by the respective countries' central governments [31, 32]. In the late 1990s, the advent of mobile telecommunications brought significant changes to the African continent, so that countries like Nigeria, Cote d'Ivoire, and Uganda had more mobile phones than fixed lines [31]. In Africa, the mobile telecommunications system has a faster and broader penetration and deployment than fixed lines, and some nations, such as the South Africa, Comoros, Uganda, Kenya, Mauritius, Malawi, and the Seychelles, have increased their rural mobile network coverage as of 2010 [33]. The advancement of mobile technology has contributed to the creation, acceptance and dissemination of mobile apps to promote mobile services, leading to the production and rise in use of different kinds of mobile devices such as smartphones.

The procurement and service of a fixed-line telephone system is highly expensive. As a result, much of the African population in particular rural dwellers and low-income earners cannot afford or have access to it [4, 32]. A broad range of network coverage is provided by mobile technology enabling quick access to the African population to telecommunications services [34, 35]. Communication services are currently accessible and affordable and more than $70 \%$ of the African people is linked to the digital telecommunications network[27]. Thus, mobile technology services enable digital inclusion.

The development, delivery and use of mobile technology services included a group of interconnected network entities called the mobile ecosystem [16, 36, 37]. The African mobile ecosystem comprises of various entities that are involve in the development and delivery of mobile innovation services including mobile network operators, sales agencies, government and other stakeholders [16, 7]. Several facets of the mobile ecosystem in conjunction with a defined system development method are involved in the process of creating, delivering, implementing and disseminating mobile apps [38]. With regard to the stakeholder's priorities, aspirations, operational services, and some predicting factors [39] mobile apps are developed for different services. There are several types of mobile apps that offer unique services to their users, including: Mobile Learning [40], Mobile Commerce [41], Mobile Health [42], Mobile Agriculture [43], Mobile Banking [44], and Mobile Governance [45] used to offer different online services respectively. In this study, these various kinds of mobile apps are categorised as the classification of mobile apps. The use of mobile apps in Africa has added tremendously to the growth of Africa [1]. That is, mobile services are rising the living standards of the people of Africa, resulting in the promotion of the economic, social, political, and educational development of all African nations. [46, 47]. Thus, the ecosystem promotes mobile apps innovations which accelerate economic growth, though there are some issues to be considered.

While mobile app developments are growing in Africa, some areas, especially rural locations, are still experiencing a slow penetration of this advanced technology due to certain factors or issues of influence [29]. Around $60 \%$ of Africans resides in remote areas 
where a large number of potential adopters are limited by the poor network connectivity, low income, unstable economic situations, and social and political instability [29]. In addition, some scholars have established numerous important factors that need to be addressed, including relative advantage, complexity, compatibility, trialability, observability, [9], [48]-[49], social influence, user perception, perceived technology reliability [49, 50], facilitating conditions [51], demographic, mobile technology self-efficacy, ICT anxiety, perceived financial cost [49, 52, 53] and culture [54], for greater acceptance and distribution of mobile apps in Africa.

However, in the African background, it is important to understand the whole procedures of adopting and disseminating mobile apps and to create a well-defined structure for this process. The goal of this study is to address these issues by developing a well-defined framework that can encourage the wider and more efficient use of home-made mobile apps, not only in the country of origin but also in other African nations. Through a survey conducted in five selected nations representing five geographical regions in Africa the process of mobile apps adoption and diffusion was examined. These countries include south (South Africa), west (Nigeria), north (Morocco), east (Kenya) and central (Democratic Republic of Congo).

These countries were selected because:

1. Each country is situated in one of Africa's five regions.

2. There is a significant population in each of the countries chosen.

3. More references were made to them in the literature review than to those in their respective regions.

4. Each region is presumed to have a similar lifestyle type.

\subsection{What is a Framework?}

A framework is a blueprint or guide consisting of principles, constructs and concept of a theory [55]. According to Fulton, Krainovich-Miller [55, 56], conceptual framework is a structure of concepts that offers the foundation for the development of an abstract idea. For this study, a framework is an abstraction for a successful mobile apps adoption and diffusion among entire African social system. This study seeks to develop a conceptual framework for mobile apps adoption and diffusion in Africa. Applying regression analysis to the research model, the relationships between the research constructs can be revealed.

\section{Related Works}

Although there is no general framework for the adoption and diffusion of mobile apps in all African geographical areas but some related works have been carried out. The existing frameworks apply to a particular category of mobile app and to a unique environment or country. A model for the adoption of mobile health in developing countries was proposed by Kiongo [13] using Kenya as a case study. In the framework, social influence, facilitating conditions, reliability, cost and simplicity are considered as determining factors of the intention to adopt and use mobile health. In a model for mobile banking adoption in Ghana, Crabbe, Standing [14] highlighted that usefulness, reliability and culture are factors shaping the attitudes and behavioural intention to use mobile banking. Bwalya and Healy [15] proposed a conceptual model for e-government adoption in the SADC region with consideration to the following factors; culture, user support, usefulness, ease of use and appropriate ICT infrastructure. Social influence, facilitating conditions, cost, culture and reliability are the predicting factors considered in a model for the adoption of e-commerce in Nigeria [57]. According to Moore and Benbasat [58], relative advantage and complexity are equivalent to usefulness and ease of use. In the light of the above-proposed frameworks, there is a need for a general model that can be applied to different domains and different regions/countries in Africa to increase the use of African-based mobile apps across all African nations.

\subsection{The Gap}

The development of mobile apps in Africa is growing rapidly [7]. A variety of mobile app start-ups have been introduced across several African countries to promote mobile app technologies. [2]. The African mobile ecosystem has promoted the creation of various kinds of mobile apps that are available on the African technology market. As a result, a large number of mobile apps originated in Africa [2]. From the literature, all the existing suggested frameworks are for specific kinds of mobile apps [13]-[15], [57] and there is no framework for the general mobile apps adoption and diffusion in Africa. In practice, most of the mobile apps created in Africa are actively used in the country where they are originated, while there is low or no acceptance of these mobile apps in other nations of the continent. It is necessary to have a common structure that will enable a wider adoption of mobile apps (especially local mobile apps) across African nations. This research will therefore assist to close the gap by suggesting an overall framework for mobile apps adoption and diffusion in Africa.

\section{Research Conceptual Model (RCM)}

Drawing from the diffusion of innovation framework, previous related studies on Africa context regarding the framework for adoption of mobile apps and their findings, a conceptual model for this study is, therefore, proposed as displayed in figure 1. The RCM demonstrates the methodological approach to accomplish the research aim. The model consists of three components including mobile apps innovations, innovation decision process and adopting unit. The mobile apps innovations component describes mobile apps and their features; innovation decision processes component deals with the acceptance, rejection, use, discontinuance and continues the use of mobile apps whereas adopting unit contains the influencing factors of mobile apps adoption and diffusion. Achieving the primary goals of this research work would validate this model.

The Mobile apps section describes that: as an innovation, a newly created mobile app should be considered to be better than the previous similar apps that it replaces, fits well with the intended potential users, easy to understand and use, tested before implemen- 


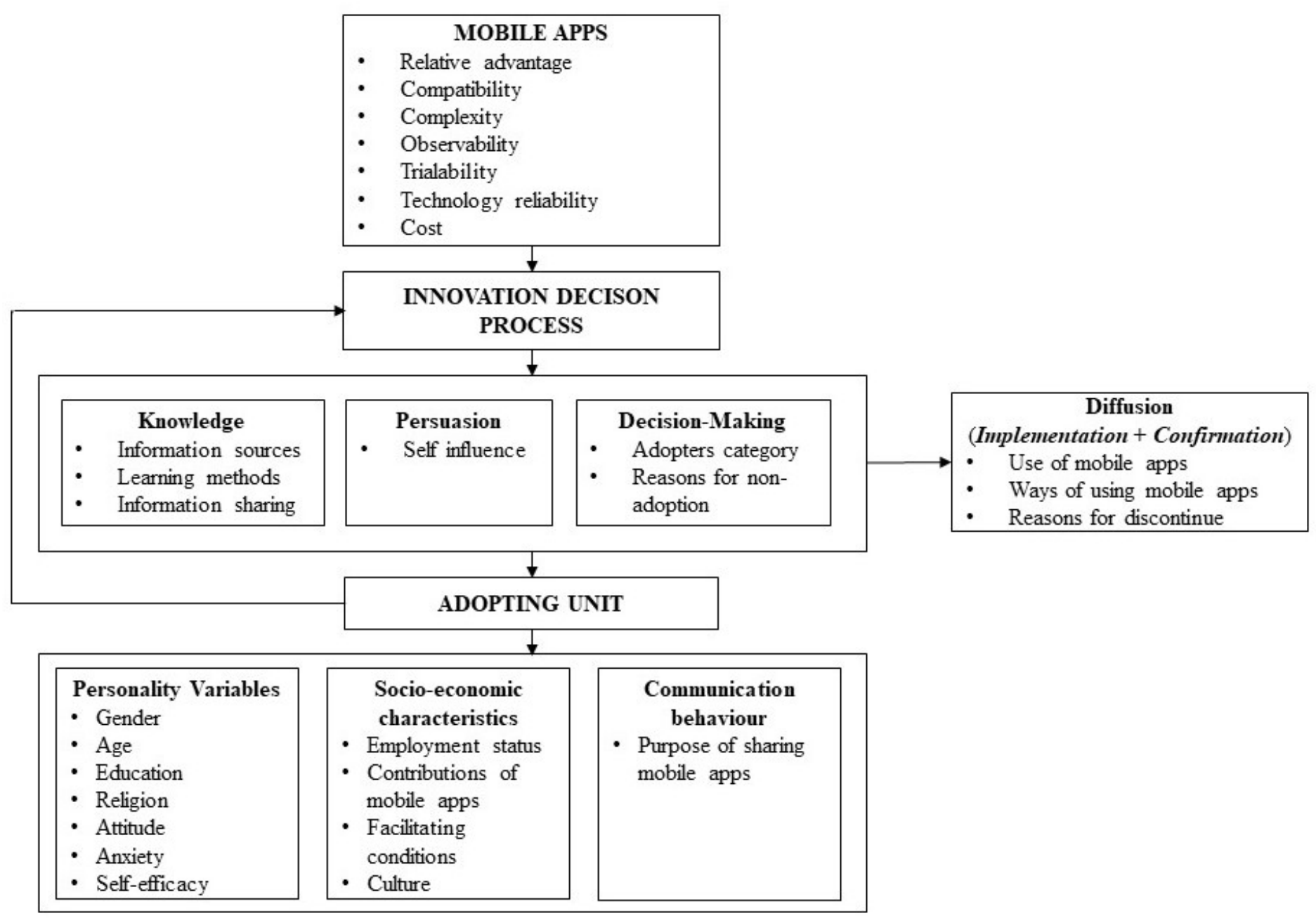

Figure 1: Research conceptual model

tation, accessible to people, and be credible. The innovation decision process explains the need for the public to be aware about the nature of a new mobile app, including source of information, the method of using the mobile app, and how to exchange information about it. Given these will help to influence the decision-making process of the adopters, either to accept or reject the mobile app. The adopting unit deals directly with the individual adopters, whereby certain personalized factors, such as personality variables, socio-economic characteristics, and communication behaviour, can affect the process of adoption. Overall, with the influence of good innovation characteristics, personalised factors and good decision-making process, a newly developed mobile app may gain widespread acceptance within the African social system.

\section{Research Methodology}

This research requires quantitative data from mobile apps users residing in five selected countries representing various African geographical regions. Hence, the positivism paradigm was adopted whereby a scientific method was used to gather self-determined data from the participants without the researcher's influence. The non-probabilistic technique of sampling was introduced since it is not easy in Africa to get the correct amount of mobile app users. In choosing the sample population for data gathering, purposive and convenience methods were considered. Purposive sampling involves the selection of a target audience based on a specific purpose. The study aims at the mobile apps adoption and diffusion and the population was selected from the area where there are large number of users of mobile apps for various activities. Convenience sampling is a particular type of data collection approach that entails the gathering of data from the participants that can be conveniently reached within the chosen population.

As the entire likely sample could not be covered by the researcher, therefore it was appropriate to pick a sample population containing all the desired participants. Thus, the purposive method was needed. The researcher was unable to reach everyone in the selected sample except those who were easily accessible; the convenience technique became essential at this juncture and was also applied. Educational institutions (universities) were selected with the purposeful method, and data was obtained from the participants with the convenience method. In order to achieve the research goals, the RCM formed the basis of the survey performed in the selected 
nations.

\subsection{The Research Instrument - The Questionnaire}

The questionnaire was used as a data collection tool. It consists of seven sections containing close-ended questions with a 5-point scale rating of 1-5 (1 strongly disagreed and 5 strongly agreed). These sections are:

1. Background information - collects individuals (participants) details.

2. Mobile applications - examines the extent of participants' awareness of mobile app technologies.

3. mobile apps innovation Adoption and diffusion - examine Africa's acceptance and use of mobile apps.

4. Innovation features - examine the participants' perceptions of the importance of the characteristics of mobile apps.

5. Impact of mobile apps - examine the contributions of mobile apps to African growth.

6. Predicting factors - examine the factors influencing the acceptance and use of mobile apps in Africa.

7. Adopter's category - to determine the types of adopters of mobile apps existing in Africa.

A pilot or experimental test was conducted to make sure that the questionnaire can resloved the important parts of the study. All the recommendations were properly considered and effected. Also, the questionnaire was vetted and approved by a Statistical Consultant. The target population is the mobile apps users from the chosen nations in Africa. In the delivery of the study questionnaires, drop off and electronic/online approaches were applied. Participants were drawn from various age groups and job status to ensure that general views are covered by the collected data. A contact person was hired to help us in collecting responses from the respondents in each of the respective counties. The person was informed of the research process and the aim of the study was clarified. A total of 1285 of the 2300 distributed questionnaires were returned, providing a response rate of $55.87 \%$. The responses comprise of 957 drop off and 328 electronically (online google forms) completed questionnaires. The responses obtained from the participants of each country was shown in Table 1

Table 1: Participants' responses from each country

\begin{tabular}{|l|c|c|}
\hline Country & Amount disseminated & Amount received \\
\hline South Africa & 500 & 335 \\
\hline Nigeria & 500 & 370 \\
\hline Morocco & 400 & 116 \\
\hline Kenya & 400 & 212 \\
\hline DRC & 500 & 252 \\
\hline Total & 2300 & 1,285 \\
\hline
\end{tabular}

Table 2: Reliability

\begin{tabular}{|l|c|c|c|}
\hline \multicolumn{1}{|c|}{ Research variables } & Mean & $\begin{array}{c}\text { Std } \\
\text { dev. }\end{array}$ & $\begin{array}{c}\text { Cronbach Alpha } \\
\text { Coefficient }\end{array}$ \\
\hline Business interactions. & 3.19 & 0.99 & 0.76 \\
\hline Individual interaction. & 3.83 & 0.86 & 0.69 \\
\hline External initiated actions. & 3.21 & 0.98 & 0.66 \\
\hline Personal initiated actions. & 3.61 & 0.99 & 0.60 \\
\hline Organizations and events. & 3.36 & 0.99 & 0.61 \\
\hline Individual activities. & 3.68 & 0.86 & 0.60 \\
\hline $\begin{array}{l}\text { Relative advantage and } \\
\text { compatibility }\end{array}$ & 3.59 & 0.84 & 0.79 \\
\hline Observability & 3.44 & 0.87 & 0.60 \\
\hline Trialability & 3.48 & 0.79 & 0.69 \\
\hline $\begin{array}{l}\text { Perceived technology } \\
\text { reliability }\end{array}$ & 3.38 & 0.83 & 0.61 \\
\hline Self-efficacy & 3.58 & 0.79 & 0.64 \\
\hline Social influence & 3.56 & 0.96 & 0.64 \\
\hline Facilitating conditions & 3.36 & 0.99 & 0.65 \\
\hline Financial cost & 3.44 & 0.98 & 0.64 \\
\hline Cultural effect & 3.31 & 0.88 & 0.63 \\
\hline $\begin{array}{l}\text { Contributions to the } \\
\text { healthcare system. }\end{array}$ & 3.52 & 0.96 & 0.75 \\
\hline $\begin{array}{l}\text { Contributions to education } \\
\text { and social activities. }\end{array}$ & 3.65 & 0.75 & 0.61 \\
\hline $\begin{array}{l}\text { Contributions to the } \\
\text { economy. }\end{array}$ & 3.62 & 0.85 & 0.61 \\
\hline Contributions to politics. & 3.60 & 0.86 & 0.60 \\
\hline Contributions to finance. & 3.79 & 0.87 & 0.61 \\
\hline Contributions to agriculture. & 3.39 & 0.95 & 0.69 \\
\hline Late Majority and Laggards & 3.25 & 0.95 & 0.63 \\
\hline Non-Adopters & 3.17 & 1.02 & 0.78 \\
\hline Innovators & 3.19 & 0.88 & 0.80 \\
\hline Early adopters & 3.33 & 0.89 & 0.72 \\
\hline Early majority & 0.88 & 0.71 \\
\hline
\end{tabular}

\section{Analysis}

To explain the frequency and response percentage of the participants, statistical analysis was performed on all the study variables. Cronbach alpha reliability analysis was used to measure reliability coefficients of the investigating variables (criteria 0.6). In order to assess the difference in the groups of dependent variables, analysis of variance (ANOVA) was conducted on the study variables, involving individual profile data (criteria, $\mathrm{p}<0.05$ ). Finally, regression analysis was performed to measure the relationship among the dependent and the independent variables. The results obtained from the data analysis will be interpreted on the basis of the RCM components. See section 7

\subsection{Reliability}

Using Cronbach alpha coefficient analysis, the reliability of the study variables was checked and it was found that the factors were internally consistent $(\alpha=0.67)$. Also, the correlation scores of all the elements are the same $(r=1)$, meaning that the factor elements 
associate with each other. The reliability results are displayed in Table 2 .

\section{Survey Results}

The main objective of this research is to develop a framework for mobile app adoption and diffusion in Africa. The quantitative data was obtained from 1,285 participants from five chosen nations representing the geographical regions of Africa. The data collected was carefully extracted, statistically analysed and the results were obtained. The outcomes of the survey are presented according to the mean values of the responses obtained from the participants with respect to scale rating except the participants' profiles which is presented on percentages.

\subsection{Participants' profiles}

The research sample population consists of $703(54.71 \%)$ of males and $582(45.29 \%)$ of females. This suggests a reasonably equal distribution of the gender categories (male and female). Mobile apps are designed to be used equally by all, regardless of gender [56]. It shows a good representation of the African population. Participants were of various age groups, from less than 20 to 70 years of age and above. The results revealed that the majority of participants were between the age groups of 20-30 and 31-40 with the proportions of $409(31.8 \%)$ and $379(29.5 \%)$ respectively. That is, $>60 \%$ of the participating population was between the ages of 20 and 40 years All levels of education, including Matrics, Diploma, University, Masters, Doctorate and others were involved in this study. 'Others' means the set of people that are deemed to be uneducated and could not fall into these classifications. $93.3 \%$ of respondents are educated, while $3.7 \%$ are uneducated. This shows that the responses were collected from individuals who had acquired some knowledge of mobile apps innovations and services. A large proportion of the participants are working class and students with 615 (47.9\%) and $402(31.3 \%)$ respectively. Such people have the capacity of embracing and using mobile apps, even though some financial costs are involved. In addition, students and workers are also more involved in many things than elderly and unemployed people, allowing more use of mobile apps for different tasks.

\subsection{Mobile apps Component}

This section investigated the participants' perceptions of the features of mobile apps.

Relative advantage - is the degree to which users of a specific mobile app considered it to be more improved than another equivalent mobile app. The mean value of the participants' responses is 3.74 meaning that relative advantage is an influencing feature of mobile apps.

Compatibility - is the extent to which mobile apps suit with the exiting values, practices and demands of the prospective adopters. The obtained mean value of the participants' responses is $3.78 \mathrm{sug}$ gesting that compatibility is a significant feature of mobile apps.

Complexity - the degree to which participants believe that the use of mobile apps is affected by how simple it is to use the mobile app. The obtained mean value of the participants' responses is 3.78 suggesting that complexity is an influencing characteristic of mobile apps.

Observability - is the degree to which participants believe that seeing the impact or what can be achieved with the mobile app will convince people to accept and use the mobile app. The obtained mean value is 3.52 suggesting that observability is an influencing feature of mobile apps.

Trialability - is the degree to which the participants believe that testing a mobile app before adopting can influence the adoption of the mobile app. The obtained mean value of the participants' responses is 3.72 suggesting that trialability is an influencing feature of mobile apps.

Perceived technology reliability - deals with the belief, danger, and credibility involved in using a new mobile app. The obtained mean value of the participants' responses is 3.38 suggesting that perceived technology reliability is an influencing characteristics of mobile apps.

Cost - are the financial costs involved with the adoption and usage of mobile app services. The obtained mean value of the participants' responses is 3.44 suggesting that cost is an influencing feature of mobile apps.

\subsection{Innovation Decision Process Component}

This section investigated the innovation decision process of mobile apps including the knowledge, persuasion, decision-making, and how the information about mobile apps are being spread in Africa. Information sources - sources of disseminating information about the existence of mobile apps. The obtained mean values are external initiated actions, 3.21 and personal initiated actions, 3.61 indicating that the sources are important to the acceptance and use of mobile apps.

Learning methods - are the methods of learning how to use mobile apps correctly. The obtained mean values from the participants' responses are: interpersonal communications, 3.6; the internet, 3.5; operational manual, 3.3; informal training, 3.3 and formal training, 2.8. This implies that all the methods are significant except formal training method.

Information sharing - are the communication channels through which the participants share the information about mobile apps with one another. The obtained mean values from the participant's responses are: organizations and events, 3.36 and personal activities, 3.68 indicating that these are significant communication channels of mobile apps adoption diffusion.

Social influence - is the level to which the adopters of mobile apps are influence by others (family, friends, government, etc.). The obtained mean value is 3.56 indicating that social influence has a significant effect on the consumers intention to adopt and use mobile apps.

Late majority and Laggards category - are the most skeptical (wait and see) and most localized set of adopters of mobile apps. The obtained mean value is 3.25 indicating a significant group of mobile apps adopters in Africa.

Non-adopters category - are the set of participants that do not adopt and use mobile apps at all. The obtained mean value from the participants' responses is 3.18 indicating that there are non-users of 
mobile services in Africa.

Innovators category - involve a group of users eager to experience or practice new innovation, regardless of the advantages or losses associated with it. The obtained mean value from the participants responses is 3.19 indicating a significant group of mobile apps adopters in Africa.

Early adopters category - they are social system leaders and advisors and their acts are confined within the limits of the social system. The obtained mean value from the participants' responses is 3.34 indicating a significant group of mobile apps adopters in Africa.

Early majority category - they are non-leaders with strong social interactions who actively promote invention and they are neither the first nor the last to adopt the innovation. The obtained mean value from the participants' responses is 3.35 indicating a significant group of mobile apps adopters in Africa.

Reasons for non-adoption - are the factors that cause the rejection of mobile apps. The obtained mean values from the responses of the participants are: mobile apps occupies a large memory space in a phone, 3.4; use of mobile apps takes time, 3.3; use of mobile apps distracts focus from other tasks, 3.3; use of mobile apps is costly, 3.3. The results indicate that these reasons may cause the rejection of mobile apps.

Use of mobile apps - Ask to decide whether participants are mobile app adopters/users. The obtained mean value from the participants' responses is 4.0 indicating that most of the sample population are mobile apps users.

Ways of using mobile apps - determine the different aims of using mobile apps. From the responses of the participants, the obtained mean values are: business use, 3.20 and individual use, 3.83 indicating that these are the important ways of using mobile apps in Africa.

Reasons for discontinuance - are the causes of stopping the use mobile apps. The obtained mean values from the participants' answers are: a new improved one to replace the existing one, 3.5; unsatisfactory results, 3.5; not easy to use, 3.3; lack of support, 3.2. The results imply that these are significant motives that may cause users to stop using a particular mobile app.

\subsection{Adopting Unit Component}

Attitude - is the reaction (positive or negative) of adopters towards new mobile apps. The obtained mean value from the the answers of the participants is 3.39 suggesting that attitude has significant effect on the acceptance and use of mobile apps.

Nervousness/anxiety - is the degree of nervousness whenever a new mobile app is introduced. The obtained mean value from the participants' responses is 3.20 suggesting that anxiety has significant effect on the acceptance and use of mobile apps.

Predicting factors - are the factors that influence mobile apps adoption and diffusion. The obtained mean values from the participants' responses are: self-efficacy, 3.58; facilitating conditions, 3.56; culture, 3.31. The results imply that these are important factors that may impact mobile apps adoption and diffusion.

Contribution of mobile apps - are the effect of the use of mobile apps services on the growth of Africa. The obtained mean values from the participants' responses are: contributions to healthcare system, 3.52; contributions to education and social activities, 3.65; contributions to economy, 3.62; contributions to politics, 3.60; contributions to finance, 3.80; contributions to agriculture, 3.40.

Purpose of information sharing - are the motives for spreading the news about mobile apps. The obtained mean values from the participants' responses are: to enable easy communications, 3.88 and to facilitate ones activities, 3.55 indicating that these are significant purposes of information sharing regarding mobile apps.

\section{Regression Analysis}

To analyse the association among the dependent and independent variables, multiple regression analysis was conducted. Two regression techniques were used to ensure that more accurate results are produced, including Enter and Stepwise techniques. Because the proposed framework measures the adoption and diffusion of mobile apps, the variables in the adoption and diffusion sections of the $\mathrm{RCM}$ are the dependent variables, while the remaining research variables are the independent variables. Regression analysis determines how the variations in the independent variables are associated to the variations in the dependents variables. These variations are represented by the coefficients while the $\mathrm{p}$-values show the significant level of the variations. $\mathrm{P}<0.05$ is the appropriate significant level. It suggests a 95\% confidence interval (the possibility of obtaining the accurate effect). Any of the variables (predictor) that has $\mathrm{p}<0.05$ is potential to be included into the model. The $\mathrm{R}^{2}$ shows the proportion of the variance accounted for by the independent variables in the dependent variable. In Table 3 , the effect sizes and the significant level of $\mathrm{R}^{2}$ are shown, according to Ellis and Steyn [59].

Table 3: Effect sizes and significant levels of $\mathrm{R}^{2}$

\begin{tabular}{|l|l|l|l|}
\hline Effect size & Effect & $\begin{array}{c}\text { Values of } \\
\mathbf{R}^{\mathbf{2}}\end{array}$ & \multicolumn{1}{|c|}{$\begin{array}{c}\text { Conclusions of } \\
\mathbf{R}^{\mathbf{2}}\end{array}$} \\
\hline$<0.15$ & Small & $<0.13$ & Non-significant \\
\hline $0.15-0.35$ & Medium & $0.13-0.25$ & Significant \\
\hline$>0.35$ & Large & $>0.25$ & Practically important \\
\hline
\end{tabular}

The dependent variables are; the Use of mobile apps, Business uses, Individual uses, and the five categories of adopters.

The independent variables are; External initiated actions, Personal initiated actions, Organizations and events, Individual activities, relative advantage and compatibility, Observability, Trialability, Perceived technology reliability, Self-efficacy, Social influence, Facilitating conditions, Cost, Cultural effect, Contributions to the healthcare system, Contributions to education and social activities, Contributions to the economy, Contributions to politics, Contributions to finance and Contributions to agriculture. Also included to the independent variables are Informal training, Operational manual, The Internet, Interpersonal communications, Good innovations and Add to development. The significant results obtained from the regression analysis performed are shown in Tables $4-11$. 


\subsection{General Use of Mobile App}

This model indicates that the $\mathrm{R}^{2}$ is 0.291 , thus, the important independent variables accounted for $29.1 \%$ of the dependent variable variance (General use of mobile apps). $\mathrm{R}^{2}>0.25$ which is practically significant. Eight variables/predictors entered into the model which accounted for $27.3 \%$ of the entire dependent variable variance. These variables are Add to development, Good innovations, Relative advantage and compatibility, Perceived technology reliability, Contributions to healthcare, Occupy phone memory, Satisfy user expectations and Social influence. Thus, $\mathrm{R}^{2}=28.2 \%$ suggesting that the variables added in this model are practically significant for the proposed framework. See Table 4

\subsection{Business Use}

It indicates that the $\mathrm{R}^{2}$ is 0.380 , thus, the important independent variables accounted for $38.0 \%$ of the dependent variable variance (Business use). $\mathrm{R}^{2}>0.25$ which is practically significant. Eighteen variables that accounted for $37.0 \%$ of the overall dependent variable variance entered the model. These variables are Enable work activities, Relative advantage and compatibility, Culture, Add to development, Anxiety, Informal training, Internet, Contributions to finance. Satisfy User expectations, Working manual, Interpersonal communications, Advantageous, Contributions to agriculture, Contributions to politics, Observability, Social influence, Contributions to educations and social activities and Enable easy communications Thus, $\mathrm{R}^{2}>25.0 \%$ showing that the variables added in this model are practically significant for the proposed framework. See Table 5

\subsection{Individual use}

This model indicates that the $\mathrm{R}^{2}$ is 0.447 , so the significant independent variables accounted for $44.7 \%$ of the dependent variable variance (Individual use). $\mathrm{R}^{2}>0.25$ which is practically significant. Also, 15 variables that accounted for $43.8 \%$ of the overall dependent variable were added into the model. These variables are Add to development, Self-efficacy, Good innovations, Trialability, Internet, Interpersonal communications, Time consuming, Contributions to finance, Tested correctly, Observability, Contributions to agriculture, Contributions to education and social activities, Individual activities, Fits well, and Contributions to healthcare. Thus, $\mathrm{R}^{2}>25.0 \%$ suggesting that the variables added in this model are practically significant for the intended framework. See Table 6

\subsection{Late Majority and Laggards}

It indicates that the $\mathrm{R}^{2}$ is 0.256 , so the important independent variables accounted for $25.6 \%$ of the dependent variable variance (Late majority and laggard). $\mathrm{R}^{2}>0.25$, which is practically significant. Also, 12 variables that accounted for $26.7 \%$ of the dependent variables variance were added into the model. These variables are Contributions to agriculture, Contributions to politics, Formal training, Used by others, Self-efficacy, Contributions to economy, Satisfy user expectations, Organizations and events, High complexity, Social influence, Perceived technology reliability, A better replacement, Occupy phone memory, and Informal training. Thus, $\mathrm{R}^{2}>25.0 \%$ suggesting that the variables added in this model are practically significant for the intended framework. See Table 7

\subsection{Non-adopters Category}

This model indicates that the $\mathrm{R}^{2}$ is 0.312 , so the significant independent variables accounted for $31.2 \%$ of the dependent variable variance (Non-adopters). $\mathrm{R}^{2}>0.25$, which is practically significant. Also, 15 variables that accounted for $30.1 \%$ of the dependent variable variance were added into the model. These variables are Financial cost, Formal training, High complexity, Use by others, Culture, Satisfy user expectations, Facilitating conditions, Lack of support, Distraction of attention, Contributions to educations and social activities, Contributions to economy, Trialability, Observability, Good innovations, and Perceived technology reliability. Thus, $\mathrm{R}^{2}>25.0 \%$ suggesting that the variables added in this model are practically significant for the intended framework. See Table 8

\subsection{Innovators Category}

It suggest that the $\mathrm{R}^{2}$ is 0.326 , thus, the important independent variables accounted for $32.6 \%$ of the dependent variable variance (Innovators). $\mathrm{R}^{2}>0.25$, indicating practically significant. Also, 14 variables that accounted for $29.7 \%$ of the overall dependent variable variance were added into the model. These variables are Relative advantage and compatibility, Informal training, Contributions to healthcare, Perceived technology reliability, Formal training, Fits well, Add to development, Facilitating conditions, High complexity, Contributions to economy, Culture, Unsatisfactory performance, Tested correctly and Satisfy user expectations. Thus, $\mathrm{R}^{2}>25.0 \%$ demonstrating that the variables added in this model are practically significant for the intended framework. See Table 9

\subsection{Early Adopters Category}

It indicates that the $\mathrm{R}^{2}$ is 0.316 , thus, the important independent variables accounted for $31.6 \%$ of the dependent variable variance (Early Adopters). $\mathrm{R}^{2}>0.25$, indicating practically significant. Also, 9 variables that accounted for $29.7 \%$ of the overall dependent variable variance were added into the model. These variables are Relative advantage and compatibility, Self-efficacy, Contributions to agriculture, Contributions to politics, Culture, Fits well, Contributions to education and social activities, Satisfy user expectations, and Facilitating conditions. Thus, $\mathrm{R}^{2}>25.0 \%$ suggesting that the variables added in this model are practically significant for the suggested framework. See Table 10

\subsection{Early Majority Category}

This model indicates that the $\mathrm{R}^{2}$ is 0.271 , thus, the important independent variables accounted for $27.1 \%$ of the dependent variable variance (Early majority). $\mathrm{R}^{2}>0.25$, which is practically significant. Also, 14 variables entered into the model which accounted for $25.7 \%$ of the total dependent variable variance. These variables are Self-efficacy, Financial cost, Contributions to education and social activities, Occupy phone memory, Informal training, Tested correctly, A better replacement, Advantageous, Contributions to 
Table 4: Model summary of the General use of mobile apps

\begin{tabular}{|c|c|c|c|c|c|c|c|c|c|}
\hline \multicolumn{10}{|c|}{ Model Summary } \\
\hline Model & $\mathbf{R}$ & R Square & Adjusted R Square & Std. Error of the Estimate & & Change : & atist & & \\
\hline \multirow{2}{*}{1} & \multirow{2}{*}{$.539 \mathrm{a}$} & \multirow{2}{*}{0,291} & \multirow{2}{*}{0,270} & \multirow{2}{*}{0,967} & R Square Change & F Change & df1 & df2 & Sig. F Change \\
\hline & & & & & 0,291 & 13,746 & 37 & 1241 & 0,000 \\
\hline
\end{tabular}

Table 5: Model summary of Business use

\begin{tabular}{|c|c|c|c|c|c|c|c|c|c|}
\hline \multicolumn{10}{|c|}{ Model Summary } \\
\hline Model & $\mathbf{R}$ & R Square & Adjusted R Square & Std. Error of the Estimate & & Change & atist & & \\
\hline \multirow{2}{*}{1} & \multirow{2}{*}{$.617 \mathrm{a}$} & \multirow{2}{*}{0,380} & \multirow{2}{*}{0,362} & \multirow{2}{*}{0,79478} & R Square Change & F Change & df1 & df2 & Sig. F Change \\
\hline & & & & & 0,380 & 20,574 & 37 & 1241 & 0,000 \\
\hline
\end{tabular}

Table 6: Model summary of Individual use

\begin{tabular}{|c|c|c|c|c|c|c|c|c|c|}
\hline \multicolumn{10}{|c|}{ Model Summary } \\
\hline Model & $\mathbf{R}$ & R Square & Adjusted R Square & Std. Error of the Estimate & \multicolumn{5}{|c|}{ Change Statistics } \\
\hline \multirow{2}{*}{1} & \multirow{2}{*}{$.669 a$} & \multirow{2}{*}{0,447} & \multirow{2}{*}{0,431} & \multirow{2}{*}{0,64418} & R Square Change & F Change & df1 & df2 & Sig. F Change \\
\hline & & & & & 0,447 & 27,126 & 37 & 1241 & 0,000 \\
\hline
\end{tabular}

Table 7: Model summary for the Late majority and Laggard category

\begin{tabular}{|c|c|c|c|c|c|c|c|c|c|}
\hline \multicolumn{10}{|c|}{ Model Summary } \\
\hline Model & $\mathbf{R}$ & R Square & Adjusted R Square & Std. Error of the Estimate & \multicolumn{5}{|c|}{ Change Statistics } \\
\hline \multirow{2}{*}{1} & \multirow{2}{*}{$.506 \mathrm{a}$} & \multirow{2}{*}{0,256} & \multirow{2}{*}{0,234} & \multirow{2}{*}{0,83069} & R Square Change & F Change & df1 & df2 & Sig. F Change \\
\hline & & & & & 0,256 & 11,555 & 37 & 1241 & 0,000 \\
\hline
\end{tabular}

Table 8: Model summary for Non-adopters category

\begin{tabular}{|c|c|c|c|c|c|c|c|c|c|}
\hline \multicolumn{10}{|c|}{ Model Summary } \\
\hline Model & $\mathbf{R}$ & R Square & Adjusted R Square & Std. Error of the Estimate & & Change s & atist & & \\
\hline \multirow{2}{*}{1} & \multirow{2}{*}{$.559 a$} & \multirow{2}{*}{0,312} & \multirow{2}{*}{0,292} & \multirow{2}{*}{0,86144} & R Square Change & F Change & df1 & df2 & Sig. F Change \\
\hline & & & & & 0,312 & 15,234 & 37 & 1241 & 0,000 \\
\hline
\end{tabular}

Table 9: Model summary for Innovators category

\begin{tabular}{|c|c|c|c|c|c|c|c|c|c|}
\hline \multicolumn{10}{|c|}{ Model Summary } \\
\hline Model & $\mathbf{R}$ & R Square & Adjusted R Square & Std. Error of the Estimate & & Change & atist & & \\
\hline \multirow{2}{*}{1} & \multirow{2}{*}{$.571 \mathrm{a}$} & \multirow{2}{*}{0,326} & \multirow{2}{*}{0,306} & & R Square Change & F Change & df1 & df2 & Sig. F Change \\
\hline & & & & 0,73808 & 0,326 & 16,203 & 37 & 1241 & 0,000 \\
\hline
\end{tabular}

Table 10: Model summary of Early adopters category

\begin{tabular}{|c|c|c|c|c|c|c|c|c|c|}
\hline \multicolumn{10}{|c|}{ Model Summary } \\
\hline Model & $\mathbf{R}$ & R Square & Adjusted R Square & Std. Error of the Estimate & & Change & atist & & \\
\hline \multirow{2}{*}{1} & \multirow{2}{*}{$.562 \mathrm{a}$} & \multirow{2}{*}{0,316} & \multirow{2}{*}{0,296} & \multirow{2}{*}{0,75119} & R Square Change & F Change & df1 & df2 & Sig. F Change \\
\hline & & & & & 0,316 & 15,521 & 37 & 1241 & 0,000 \\
\hline
\end{tabular}

Table 11: Model summary of Early majority category

\begin{tabular}{|c|c|c|c|c|c|c|c|c|c|}
\hline \multicolumn{10}{|c|}{ Model Summary } \\
\hline Model & $\mathbf{R}$ & R Square & Adjusted R Square & Std. Error of the Estimate & & Change $s$ & atist & & \\
\hline \multirow{2}{*}{1} & \multirow{2}{*}{$.509 a$} & \multirow{2}{*}{0,271} & \multirow{2}{*}{0,237} & \multirow{2}{*}{0,76663} & R Square Change & F Change & df1 & df2 & Sig. F Change \\
\hline & & & & & 0,259 & 11,741 & 37 & 1241 & 0,000 \\
\hline
\end{tabular}


politics, Internet, External initiated actions, Culture, Satisfy user expectations and Trialability. Thus, $\mathrm{R}^{2}>25.0 \%$ suggesting that the variables added in this model are practically significant for the proposed framework. See Table 11

\section{ANOVA Test}

In order to examine the correlation among the profiles of the participants and the dependent variables, the ANOVA test was used It helps to assess the impact or statistical importance of the participants' age, educational level, employment position and religion on the acceptance and distribution of mobile apps in Africa. The ANOVA results obtained are depicted in the Table 12 with regard to the five selected countries. This suggests that with the exception of innovators and early adopters, age has relevant impact on all the dependent variables. Education level indicated an important effect on Business use and Individual use. Employment status showed an important influence on business use and non-adopter's category while religion has a substantial impact on business use, individual use, non-adopter's and innovators categories concerning the acceptance and use of mobile apps within the African social system.

\section{Interpretations and Validation of the Proposed Framework}

The RCM was developed and used in this research as the basic principles. The model entails three parts or components, which include:

1. Mobile apps Innovations,

2. Innovation decision process, and

\section{Adopting unit.}

Each of these part contains a variety of factors that constituted the study variables. Various statistical analyses were conducted to measure the importance of the study variables from the perspectives of the participants. The obtained mean values from the responses of the participants show that the research variables are essential for mobile apps to be adopted and disseminated in Africa. With regard to the proposed framework, several analyses were also, carried out to confirm if the variable fits well in the model.

To examine the relationship between the model variables, multiple linear regression analysis was carried out. For dependent variables, eight factors were considered, including the Use of Mobile apps, Business uses, and Individual uses, Late majority and Laggards, Non-adopters, Innovators, Early adopters, and Early majority. These variables are derived form the adoption and diffusion component of the model. Regarding independent variables, the remaining factors were considered. The association among the variables was tested by regression analysis. After the analysis, the eight dependent variables resulted in the eight models. These models accounted over $25.0 \%$ of the variance $\left(\mathrm{R}^{2}>25.0 \%<50.0 \%\right)$. With regards to the criteria for significant association namely the $\mathrm{p}$-values, $\mathrm{R}^{2}$, normality, tolerance and VIF, the output from regression analysis on the test of the relationships between the dependent and independent variables are interpreted below.

Model for the general use of mobile apps: $R^{2}=0.273$ suggests that the model is practically significant to the general use of mobile apps. This means that the model explained $27.3 \%$ of the variance concerning the general use of mobile apps in Africa. The model included a total of eight positive predictors: Add to development, Good innovations, Relative advantage and compatibility, Perceived technology reliability, Contributions to health care, Occupies phone memory, Satisfies user expectations and Social influence. All these variables have a positive impact on Africa's overall use of mobile apps.

The model therefore suggests that the dependent variable (general use of mobile apps) increases as predictive variables increase. this implies that that the adopters' attitude towards adoption and use of mobile apps can be shaped by good mobile apps that can contribute to Africa's development. Competitive benefit and usability increase the opportunity for mobile apps to be adopted and used. Safety assurance and decreased risk of using mobile apps, as well as the conviction of close friends and family increase the general use of mobile apps, even though the use of mobile apps takes up a great portion of phone storage.

Model for Business use: $\mathrm{R}^{2}=0.370$ suggests that the model is practically significant to the use of mobile apps for business purposes. This means that the model explained $37.0 \%$ of the variance in the use of mobile apps in Africa for business purposes. In the model, 14 predictors, namely Enables work activities, Relative advantage and compatibility, Culture, Adds to development, Anxiety, Informal training, Internet, Satisfies user expectations, Working manual, Interpersonal communication, Contributions to agriculture, Observability, Social influence and Enables easy communication have a positive relationship with the use of mobile apps for business purposes, while four predictors, Contributions to finance, Advantageous, Contributions to politics, and Contributions to education and social activities have a negative relationship with the use of mobile apps for business purposes.

This means that the use of mobile apps for business purposes requires mobile apps to be good, beneficial and business-friendly in order to improve their meaningful business activities. Enables work activities and easy communications improve the use of mobile apps for business interactions. Organizations, such as banks are sharing the information about their mobile banking apps and other service-related mobile apps, such as FNB eWallet with their clients to improve their services. Increasing these predictors will increase the rate of adoption and diffusion of these mobile apps meant for business purposes.

African countries are developing nations, and developing mobile apps with local content containing social behaviour and norms will attract more adopters, especially rural dwellers and the less educated. Individuals can easily observe mobile apps created with local content, such as local language, and be convinced to use them. M-commerce apps in a local language can easily be adopted and used by people in remote areas to improve their lives in that area. Interestingly, anxiety about the use of mobile apps for business purposes has been positive. It is possible because most mobile app users were nervous when using mobile apps, especially for business transactions. Using the banking apps, as well as M-commerce apps 
Table 12: ANOVA on Participants' profiles

\begin{tabular}{|l|c|c|c|c|}
\hline \multirow{2}{*}{\multicolumn{1}{c|}{ Variables }} & \multicolumn{4}{c|}{ p-values (ANOVA) } \\
\cline { 2 - 5 } & Age & Education level & Employment status & Religion \\
\hline Use of mobile apps & 0.023 & 0.598 & 0.442 & 0.066 \\
\hline Business use & 0.000 & 0.000 & 0.000 & 0.000 \\
\hline Individual use & 0.000 & 0.000 & 0.227 & 0.022 \\
\hline Late majority and laggards & 0.000 & 0.746 & 0.990 & 0.264 \\
\hline Non-adopters & 0.000 & 0.080 & 0.004 & 0.012 \\
\hline Innovators & 0.176 & 0.835 & 0.777 & 0.023 \\
\hline Early adopters & 0.063 & 0.607 & 0.197 & 0.065 \\
\hline Early majority & 0.010 & 0.110 & 0.572 & 0.313 \\
\hline
\end{tabular}

attracts some degree of fear and nervousness, but they facilitate easy, quick and comfortable transactions. Although people are anxious about using such apps, the number of users increases rapidly. Users need to learn how to use mobile apps adequately for business. Increasing the learning methods of how to use mobile apps will improve the adoption and diffusion of mobile apps. Unfortunately, the use of mobile apps for business is not profitable, and it attracts some costs. The more banking apps you use to carry out banking transactions, the more fees you pay. The use of mobile apps for business is also not affected by political, educational and social advancement.

Model for Individual use: $\mathrm{R}^{2}=0.438$ suggests that the model is practically significant to the individual use of mobile apps. This shows that the model explained $43.8 \%$ of variance for individual use of mobile apps in Africa. The model included a total of 15 predictors, including 13 positive predictors (Adds to development, Self-efficacy, Good innovations, Trialability, Internet, Interpersonal communication, Time-consuming, Contributions to finance, Tested correctly, Observability, Contributions to education and social activities, Fits well, and Individual activities) and two negative predictors (Contributions to agriculture and Contributions to health care). Positive predictors have a positive relationship with the individual use of mobile apps, while negative predictors have a negative relationship with the use of mobile apps for individual purposes.

The model suggests that the attitude of mobile app adopters and their use thereof can be shaped by good mobile apps that can contribute to the development in Africa. The advantages of using mobile apps, including financial, educational and social benefits, affect the adoption and diffusion of mobile apps for individual purposes. The benefits of mobile social networking apps, for example have improved the adoption and use of mobile apps by individuals. Individual use of mobile apps involves the technical knowledge of how to use mobile technology, and the trial of new technology, as well as seeing others using it increases the adoption and diffusion of mobile apps. Users need to learn how to use mobile apps. The availability of learning methods, such as the Internet and interpersonal communication increase the rate of adoption and diffusion of mobile apps for individual use. The model also shows that, due to time and costs involved, there is less interest in using mobile apps to perform agricultural and health activities. In other words, the time and cost involved in using mobile apps reduces mobile app adoption and dissemination in Africa.

Model for Late majority and Laggards category: $R^{2}=0.265$ indicates that the model is practically significant to the Late and Laggards category of mobile app adopters. It implies that 26.5\% of the variance can be explained by the adopters of mobile apps in Africa's Late majority and Laggards category model. The model included a total of 12 predictors, including nine positive (Contributions to agriculture, Contributions to politics, Formal training, Used by others, Self-efficacy, Contributions to the economy, Organizations and events, High complexity, Perceived technology reliability, Occupies phone memory, and Informal training) and three negative predictors (A better replacement, Satisfies user expectations and Social influence). Any increase in positive predictors leads to an increase in the Late majority and Laggards category, while any increase in negative predictors leads to a reduction in the category of the Late majority and Laggards adopters in Africa.

The Late majority and Laggards category of adopters is the set of adopters that is sceptical about adopting new mobile apps and they adopt a "wait and see" attitude towards adopting new technology. For this category to adopt and use mobile apps, they need proper information sources, such as organization and events, as well as seeing others using them and knowing the benefits of using mobile apps. In addition, they require training and reliability assurance on the adoption and diffusion of mobile apps. Since they are the local set in the social system, they become more discouraged by their close peers and are more afraid to adopt new mobile apps.

Model for Non-adopters category: $R^{2}=0.301$ indicates that the model is practically significant to the non-adopters' category of mobile apps adopters. This means that the model explained $30.1 \%$ of the variance. The model included a total of 15 significant predictors, containing 11 positive (Financial cost, Formal training, High complexity, Use by others, Culture, Facilitating conditions, Lack of support, Distraction of attention, Contributions to the economy, Observability, and Perceived technology reliability) and four negative predictors (Good innovations, Trialability, Satisfies user expectations and Contributions to education and social activities). The 11 predictors have a positive relationship with the category of non-adopters, while the four negative predictors have a negative relationship with the category of mobile app users in Africa who are not adopters.

The model explained that the high complexity, lack of support and the financial cost involved in the adoption and diffusion of mobile apps cause some adopters in the social system of Africa not to adopt and use mobile apps. The cultural beliefs, as well as the extent of support from government and other stakeholders, and dis- 
traction from other activities increase the existence of non-adopters in Africa. It is expected that the perception of mobile apps as good innovations will change the attitudes of non-adopters. Providing a trial opportunity, satisfying users' expectations and the benefits of the use of mobile apps, especially in education and social activities, will reduce the number of non-adopters in Africa.

Model for Innovators category: $\mathrm{R}^{2}=0.317$ indicates that the model is practically significant to the innovators' category of mobile apps adopters. It implies that the innovator category model explained $31.7 \%$ of the variance. The model is related to 14 important variables/predictors, including 12 positive variables (Relative advantage and compatibility, Informal training, Contributions to health care, Perceived technology reliability, Formal training, Fits well, Facilitating conditions, Contributions to the economy, Culture, Unsatisfactory performance, Tested correctly and Satisfies user expectations) and two negative variables (Adds to development and High complexity). The positive variables increase the category of innovators as they increase, while the negative variables reduce the category as they increase.

The model explained that the challenge of competitive advantage, availability of necessary environment from government and other stakeholders, as well as trying to satisfy the consumers' demands results in the increase of the innovators' category of adopters in Africa. The advancement of technology increases users' experience and requirements, thus there is a need for innovators to create mobile apps that will suit the new improvements. For purposes of improving the economy, poor performance of mobile apps and social behaviour, more improved mobile apps are needed. This encourages or raises more innovators in the social system. On the other hand, the higher the development progress and higher complication of mobile apps in Africa, the lesser innovators are required.

Model for Early adopters category: R $2=0.297$ suggests that the model is practically significant to the early adopters' category of mobile app adopters. It means that the model described $29.7 \%$ of the variance. The model is connected to nine predictors, including eight positive predictors (Relative advantage and compatibility, Self-efficacy, Contributions to agriculture, Contributions to politics, Culture, Fits well, Contributions to education and social activities and Satisfies user expectations) and one negative predictor (Facilitating conditions). Any rise in positive predictors leads to an increase in the early adopt category, while any increase in negative predictors leads to a decrease in the early adopt category.

The model shows that the ability to use a mobile app with advanced features will be of good interest and increase the potential adopters in the early adopter's category. In addition, the ability to use mobile apps to conduct educational, agricultural and political activities, as well as to fulfill consumer needs, is increasing the category of early adopters. Conversely, the availability of a suitable setting decreases the size of the group of early adopters.

Model for the Early majority category: $R^{2}=0.257$ shows that the model is practically important for mobile apps adopters in the early majority category. This means that $25.7 \%$ of the variance was explained by the model. The model is associated with 14 predictors, including 11 positive (Self-efficacy, Financial cost, Contributions to education and social activities, Occupies phone memory, Informal training, Tested correctly, Advantageous, Contributions to politics, Internet, Culture, and Trialability) and three negative predictors (A better replacement, External initiated actions, and Satisfies user expectations). A rise in positive predictors results to an increase to the size of early majority adopter category while an increase in negative predictors reduces the size of the category.

This shows that testing a new mobile app and finding it useful will increase the interest of consumers to embrace and use the mobile app without considering the impact on the phone's memory. The availability of methods of learning, such as the Internet and informal training, increases the user's skill and knowledge. The creation of better mobile apps and the acquisition of information from external sources, such as TV and radio, on the other hand increases consumer demand, thereby decreasing the acceptance of prior mobile apps.

Regarding the personality variables (age, education level, employment status and religion), ANOVA was conducted to examine the association among personality variables and the dependable variables. The findings showed that age has a statistically significant impact on all dependent variables, with the exception of the categories of Innovators and Early Adopters. The level of education showed a major effect on business and individual uses. A significant impact from the employment status was noticed on the business use and the non-adopters categories. Concerning religion, the impact was much on business use, individual use, non-adopters' and innovators' categories. Therefore, age, level of education, employment status and religion have an important impact on the acceptance and diffusion of mobile apps in Africa in various ways.

To explore the influence of gender on the acceptance and use of mobile apps, a t-test study was carried out. No noticeable effect was shown by the result. There are no variances among males and females in the adoption and delivery of mobile apps. Mobile apps are created for everyone regardless of the gender.

Overall, various substantial portions of the variance were explained by the eight models and all $\mathrm{R}^{2}$ scores were over 25.0 points, $\left(\mathrm{R}^{2}>25.0 \%<50.0 \%\right)$. This demonstrates that these models are practically important for the adoption and diffusion of mobile applications in Africa, and all the factors/predictors of the RCM can be applied to the proposed system. Some personality profiles have also been shown to have an effect on the adoption and distribution of mobile apps in Africa, including age, education, employment status and religion. As a consequence, a correlation occurs among them and the dependent variables. The association among the study variables typically shows that they are valuable predictors of mobile apps adoption and diffusion in Africa.

\subsection{The Framework}

Based on the findings obtained from various statistical studies and recommendations, Figure 2 presents the final proposed framework for the effective adoption and diffusion of mobile apps in Africa.

\section{Conclusions and Future Work}

\subsection{Conclusion}

This research aims to create a general framework for mobile apps adoption and diffusion in Africa. A three components model was conceptualized consisting of the research variables. The positivism 


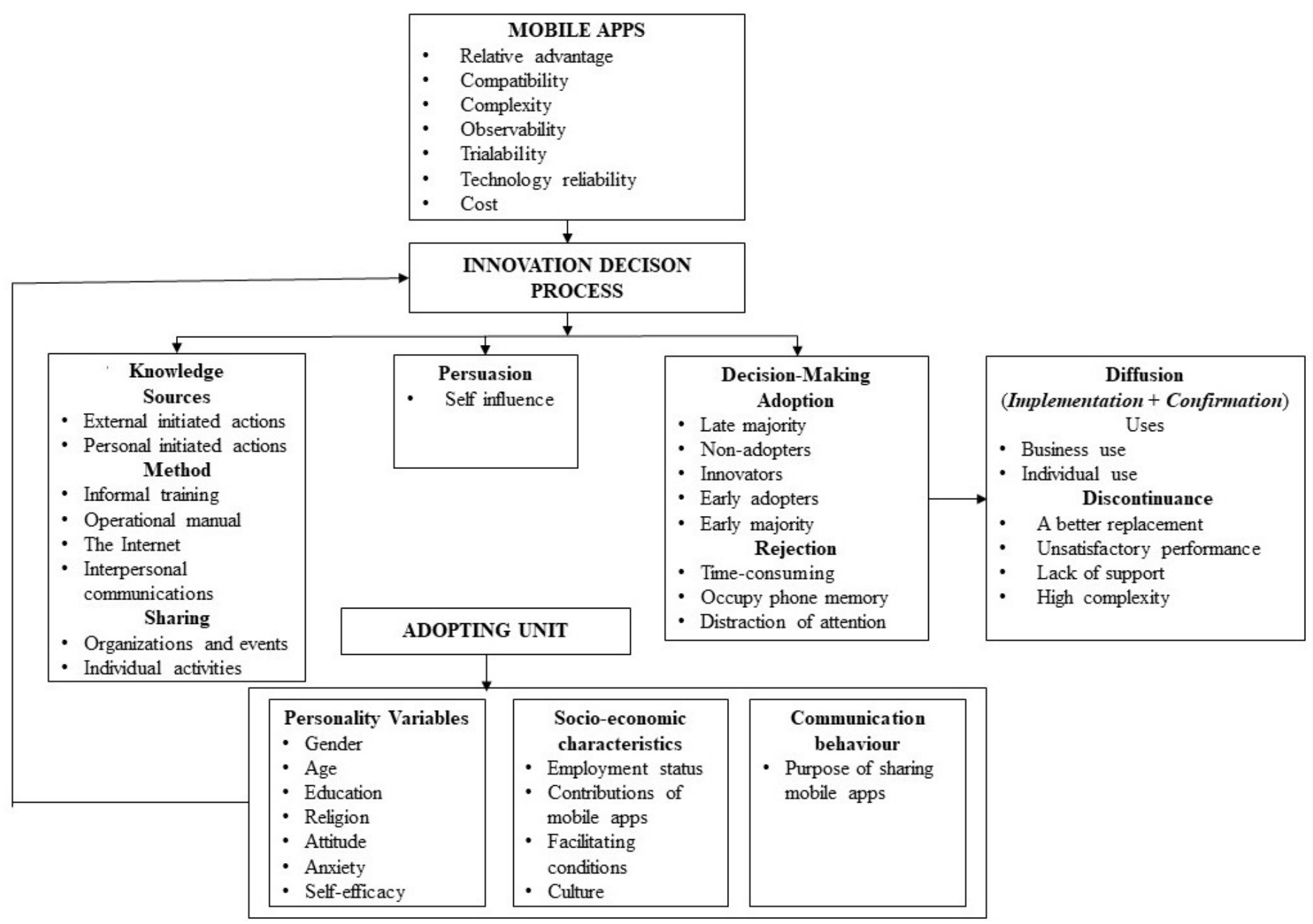

Figure 2: Validated Proposed Framework

research approach was applied. Through a survey, quantitative data were collected from the mobile apps users living in the five distinct nations representing the various regions in Africa. Various statistics analyses were carried out and the results show that the participants perceived these variables to be significant factors for mobile apps adoption and diffusion in Africa. Furthermore, a regression analysis was conducted to measure the relationships among these variables in association with mobile apps adoption and diffusion. The variable in the adoption and diffusion component of the RCM are the dependable variables whereas the remaining variables are the independent variables. The findings indicated that variables (predictors) explained more than $25.0 \%$ of the variance $\left(\mathrm{R}^{2}>25.0 \%<50.0 \%\right)$ with appropriate level of significance $(\mathrm{p}<0.05)$. These results suggest that the research variables are practically significant and they are potential or meaningful predictors of mobile apps adoption and diffusion in Africa. Thus, the variables fits well in the proposed framework. Also, the study revealed that some personality profiles such as age, education level, employment status , and religion have certain degree of influences on mobile apps adoption and diffusion in Africa. Therefore, there is a relationship among the personality variables and the dependent variables. Overall, the relationships existing among the research variables and their significant impact on mobile apps adoption and diffusion suggest that they are potential predictors of mobile apps adoption and diffusion in Africa. Consequently, the study proposed a three components framework for mobile apps adoption and diffusion in Africa.

\subsection{Limitations}

Africa is a region made up of multilingual nations and a variety of political challenges. Language gaps, cultural disparities and political uncertainty in most nations posed some serious limitations to this research work especially in Kenya, Morocco, and DRC. Also, due insufficient funding, it was not possible to reach all target audiences, particularly some rural dwellers, instead the convenient participants were used.

\subsection{Future work}

African nations are growing with potential population that are ready to embrace and use mobile technology innovations such as mobile apps. In order to further encourage mobile technology adoption, further research is needed on information awareness, the expansion of mobile services to unconnected areas, and how to bridge the gap 
between educated and uneducated users of mobile apps in Africa.

\section{References}

[1] J. Hellström, P.-E. Tröften, The innovative use of mobile applications in East Africa, Swedish international development cooperation agency (Sida), 2010. https://www.sida.se/contentassets/ b55f10e43c874c9da41203d991a7eaa0/14993.pdf.

[2] GSMA, The mobile economy Sub-Saharan Africa 2015., GSMA Intelligence. Regional report. https://gsmaintelligence. com/research. Accessed on 23/03/2016., 2015.

[3] R. Nickerson, M. Austreich, J. Eng, "Mobile technology and smartphone apps: A Diffusion of innovations analysis," in 20th Americas Conference on Information Systems, AMCIS 2014, 2014, 1-12.

[4] G. Van Zyl, Why Kenya's fixed line market is shrinking., itweb. https: //itweb.africa/content/KjlyrvwdnK57k6am. Accessed on 24/06/2016., 2013.

[5] S. Lee, M. Marcu, S. Lee, "An empirical analysis of fixed and mobile broadband diffusion," Information Economics and Policy, 23(3-4), 227-233, 2011, doi:10.1016/j.infoecopol.2011.05.001.

[6] A. A. Shaikh, H. Karjaluoto, "Mobile banking adoption: A literature review," Telematics and informatics, 32(1), 129-142, 2015, doi:10.1016/j.tele.2014.05. 003 .

[7] S. Murugesan, "Mobile apps in Africa," IT Professional, 15(5), 8-11, 2013, doi:10.1109/MITP.2013.83.

[8] C. W. Okonkwo, M. Huisman, E. Taylor, "An investigation on the adoption and diffusion of mobile apps in Africa." Thesis submitted in fulfilment of the requirements for the degree Doctor of Philosophy in Computer Science at the North-West University, Potchefstroom campus, South Africa., 2019., 2019.

[9] E. Rogers, Diffusion of Innovations (5th ed)., New York: Free Press., 2003.

[10] I. Lule, T. K. Omwansa, T. M. Waema, "Application of technology acceptance model (TAM) in m-banking adoption in Kenya." International Journal of Computing \& ICT Research, 6(1), 2012.

[11] D. G. Taylor, T. A. Voelker, I. Pentina, "Mobile application adoption by young adults: A social network perspective," 2011. http://digitalcommons. sacredheart.edu/wcob_fac/1.

[12] D. W. Stacks, M. B. Salwen, An integrated approach to communication theory and research, Routledge, 2014.

[13] N. Kiongo, "A framework for mobile health adoption in developing countries: Case study Kenya," A framework for mobile health adoption in developing countries: Case study Kenya, 2014. http://hdl.handle.net/11295/ 74353.

[14] M. Crabbe, C. Standing, S. Standing, H. Karjaluoto, "An adoption model for mobile banking in Ghana," International Journal of Mobile Communications, 7(5), 515-543, 2009. DOI: https://doi .org/10.1504/IJMC. 2009. 024391.

[15] K. J. Bwalya, M. Healy, "Harnessing e-government adoption in the SADC region: a conceptual underpinning," Electronic Journal of E-government, 8(1), 23, 2010.

[16] C. Z. Qiang, S. C. Kuek, A. Dymond, S. Esselaar, "Mobile applications for agriculture and rural development," 2012. http://hdl.handle.net/10986/ 21892.

[17] K. S. McNamara, "Mobile applications in agriculture and rural development: Framing the Topic, and Learning from Experience," in World Bank Workshop on Mobile Innovations for Social and Economic Transformation, 2009.

[18] M. O. M. El-Hussein, J. C. Cronje, "Defining mobile learning in the higher education landscape," Journal of Educational Technology \& Society, 13(3), 12-21, 2010. https://www.jstor.org/stable/jeductechsoci.13.3.12.
[19] C. Perera, A. Zaslavsky, P. Christen, D. Georgakopoulos, "Context aware computing for the internet of things: A survey," IEEE communications surveys \& tutorials, 16(1), 414-454, 2013, doi:10.1109/SURV.2013.042313.00197.

[20] E. Oriwoh, P. Sant, G. Epiphaniou, "Guidelines for internet of things deployment approaches-the thing commandments," Procedia Computer Science, 21, 122-131, 2013, doi:10.1016/j.procs.2013.09.018.

[21] A. Giessmann, K. Stanoevska-Slabeva, B. De Visser, "Mobile enterprise applications-current state and future directions," in 2012 45th Hawaii International Conference on System Sciences, 1363-1372, IEEE, 2012. https: //doi.org/10.1109/HICSS.2012.435

[22] H. T. Dinh, C. Lee, D. Niyato, P. Wang, "A survey of mobile cloud computing: architecture, applications, and approaches," Wireless communications and mobile computing, 13(18), 1587-1611, 2013, doi:10.1002/wcm.1203.

[23] J. Donner, C. A. Tellez, "Mobile banking and economic development: Linking adoption, impact, and use," Asian journal of communication, 18(4), 318-332, 2008, doi:10.1080/01292980802344190.

[24] C. W. Okonkwo, M. Huisman, E. Taylor, "The adoption of mcommerce applications: rural dwellers perspectives," in 12th, IADIS, International conference. Information systems., pages:99-106, 2019. DOI: 10.33965/is2019_201905L013.

[25] C. W. Okonkwo, M. Huisman, E. Taylor, "Socio-Economic Contributions of Mobile Applications in Africa: Impact of Local Mobile Applications," in 2019 International Multidisciplinary Information Technology and Engineering Conference (IMITEC), 1-8, IEEE, 2019. DOI: https://doi.org/10.1109/ IMITEC45504.2019.9015912

[26] G. Intelligence, “The Mobile Economy. 2020,” 2020.

[27] G. Association, et al., "The mobile economy 2018," London: GSM Association, 2018.

[28] L. Hosman, E. Fife, "The use of mobile phones for development in Africa: Topdown-meets-bottom-up partnering," The Journal of Community Informatics, 8(3), 2012

[29] G. Association, et al., "The Mobile Economy Sub-Saharan Africa," London: GSM Association, 2017.

[30] B. Simon, "A History of Mobile Application Development," https://manifesto.co.uk/history-mobile-application-development/. Accesses on 23/06/2016. 2014., 2017.

[31] I. T. Union, World Telecommunication/ICT Development Report, International Telecommunication Union, 2006.

[32] H. K. Chavula, "Telecommunications development and economic growth in Africa," Information Technology for Development, 19(1), 5-23, 2013 https://doi.org/10.1080/02681102.2012.694794

[33] M. E. Batuo, "The role of telecommunications infrastructure in the regional economic growth of Africa," The Journal of Developing Areas, 49(1), 313-330, 2015. https://www.jstor.org/stable/24241299

[34] S. Etzo, G. Collender, "The mobile phone 'revolution'in Africa: rhetoric or reality?" African affairs, 109(437), 659-668, 2010. DOI: https://doi .org/ 10.1093/afraf/adq045

[35] S. H. Lee, J. Levendis, L. Gutierrez, "Telecommunications and economic growth: an empirical analysis of sub-Saharan Africa," Applied Economics, 44(4), 461-469, 2012. DOI: https://doi.org/10.1080/00036846.2010 508730

[36] J. Zhang, X.-J. Liang, "Business ecosystem strategies of mobile network operators in the 3G era: The case of China Mobile," Telecommunications policy, 35(2), 156-171, 2011. DOI: https://doi.org/10.1016/j.telpol.2010 12.009

[37] R. Lindgren, O. Eriksson, K. Lyytinen, "Managing identity tensions during mobile ecosystem evolution," Journal of Information Technology, 30(3), 229-244, 2015. DOI: https://doi.org/10.1057/jit.2015.8 
[38] W. C. Okonkwo, M. Huisman, "The use of system development methodologies in the development of mobile applications: Are they worthy of use?" in 2018 IEEE 42nd Annual Computer Software and Applications Conference (COMPSAC), 2, 278-283, IEEE, 2018. DOI: https: // doi.org/10.1109/COMPSAC. 2018.10243.

[39] C. W. Okonkwo, M. Huisman, "The predicting success factors of mobile applications development: organizational perspectives," in 12th, IADIS, International conference. Information systems., 11-18, 2019. DOI: 10.33965/is2019_201905LO02.

[40] W.-H. Wu, Y.-C. J. Wu, C.-Y. Chen, H.-Y. Kao, C.-H. Lin, S.-H. Huang, "Review of trends from mobile learning studies: A meta-analysis," Computers \& Education, 59(2), 817-827, 2012. DOI: https://doi.org/10.1016/j. compedu.2012.03.016.

[41] S. Okazaki, F. Mendez, "Exploring convenience in mobile commerce: Moderating effects of gender," Computers in Human Behavior, 29(3), 1234-1242, 2013. DOI: https://doi.org/10.1016/j.chb.2012.10.019.

[42] C. Free, G. Phillips, L. Watson, L. Galli, L. Felix, P. Edwards, V. Pa-tel, A. Haines, "The effectiveness of mobile-health technologies to improve health care service delivery processes: a systematic review and meta-analysis," PLoS medicine, 10(1), e1001363, 2013. DOI: https://doi .org/10.1371/ journal.pmed. 1001363 .

[43] A. Gichamba, I. A. Lukandu, "A model for designing M-agriculture applications for dairy farming," The African Journal of Information Systems, 4(4), 1, 2012. https://digitalcommons. kennesaw.edu/ajis/vol4/iss4/1.

[44] P. Van der Boor, P. Oliveira, F. Veloso, "Users as innovators in develop-ing countries: The global sources of innovation and diffusion in mobile banking services," Research Policy, 43(9), 1594-1607, 2014. DOI: https : // doi.org/10.1016/j.respol.2014.05.003.

[45] J. Hellström, "Mobile Governance: Applications, Challenges and Scalingup in Poblet, M.(Ed.),"Mobile Technologies for Conflict Management: Online Dispute Resolution, Governance, Participation"," 2012. DOI: https:|/doi.org/10.1007/978-94-007-1384-0_12.

[46] D. Baelden, L. Van Audenhove, "Participative ICT4D and living lab research: The case study of a mobile social media application in a rural Tanzanian University setting," Telematics and Informatics, 32(4), 842-852, 2015. DOI: https://doi.org/10.1016/j.tele.2015.04.012.

[47] J. C. Aker, I. M. Mbiti, "Mobile phones and economic development in Africa," Journal of economic Perspectives, 24(3), 207-32, 2010. DOI: 10.1257/jep.24.3.207.

[48] S. Tunmibi, A. Aregbesola, E. Asani, "Factors influencing the adoption of smart phones by university students-A cross-border approach," African Journal of Computing \& ICT, 8(1), 1-16, 2015.
[49] B. M. Achieng, B. K. Ingari, "Factors influencing the adoption of mobile banking in Kenya's commercial banks: A case of Kenya Commercial Bank (KCB) Kilindini branch," International Journal of Scientific and Research Publications, 5(10), 1-14, 2015.

[50] R. A. Oluoch, Factor affecting adoption of mobile banking technology in Kenya: A case of bank customers within Nakuru Municipality, Ph.D. thesis, 2012.

[51] J. Mtebe, R. Raisamo, "Investigating students' behavioural intention to adopt and use mobile learning in higher education in East Africa," International Journal of Education and Development using ICT, 10(3), 2014 https://www.learntechlib.org/p/148476/

[52] W. P. Mtega, R. Bernard, A. C. Msungu, R. Sanare, "Using mobile phones for teaching and learning purposes in higher learning institutions: The case of Sokoine University of Agriculture in Tanzania," 2012. https://repository. ubuntunet.net/handle/10.20374/130

[53] F. Iddris, "Barriers to adoption of mobile banking: Evidence from Ghana," International Journal of Academic Research in Business and Social Sciences, 3(7), 356-370, 2013. DOI: https://doi.org/10.6007/IJARBSS/v3-i7/59

[54] F. O. Bankole, O. O. Bankole, I. Brown, "Mobile banking adoption in Nigeria," The Electronic Journal of Information Systems in Developing Countries, 47(1), 1-23, 2011. DOI: https://doi.org/10.1002/j.1681-4835.2011. tb00330.x

[55] A. Osanloo, C. Grant, "Understanding, selecting, and integrating a theoretical framework in dissertation research: Creating the blueprint for your "house"," Administrative issues journal: connecting education, practice, and research, 4(2), 7, 2016. DOI: 10.5929/2014.4.2.9.

[56] S. Fulton, B. Krainovich-Miller, C. Cameron, "Finding and appraising the literature," Nursing Research in Canada-E-Book: Methods, Critical Appraisal, and Utilization, 90, 2014.

[57] S. Chiemeke, A. Evwiekpaefe, "A conceptual framework of a modified unified theory of acceptance and use of technology (UTAUT) Model with Nigerian factors in E-commerce adoption," Educational Research, 2(12), 1719-1726, 2011.http://www. interesjournals.org/ER

[58] G. C. Moore, I. Benbasat, "Development of an instrument to measure the perceptions of adopting an information technology innovation," Information systems research, 2(3), 192-222, 1991. DOI:https://doi.org/10.1287/ isre.2.3.192

[59] S. Ellis, H. Steyn, "Practical significance (effect sizes) versus or in combination with statistical significance (p-values): research note," Management dynamics: journal of the southern african institute for management scientists, 12(4), 51-53, 2003. 